\title{
$\underline{\mathbf{P}-131}$
}

\section{Comparison of Microwave-Assisted Extraction and Hydrodistilation Method in the Extraction of Essential Oils from Aquilaria malaccensis (Agarwood) Oil}

\author{
Norfatirah $\mathrm{MS}^{1}$, Tajuddin $\mathrm{SN}^{1,{ }^{*}}$, Chemat $\mathrm{F}^{2}$, Rajan $\mathrm{J}^{1}$ and Yusoff $\mathrm{MM}^{1}$ \\ ${ }^{1}$ Faculty of Industrial Sciences and Technology, Universiti Malaysia Pahang, Lebuhraya Tun, Razak, 26300 Gambang, \\ Pahang, Malaysia; ${ }^{2}$ Université d'Avignon et des Pays de Vaucluse, INRA, UMR408, F-84000 Avignon, France
}

Aquilaria malaccensis (Thymelaeaceae) is a type of agarwood, mainly found in Malaysia. Microwave-assisted extraction (MAE) method, a new technique to extract essential oil from agarwood, was used and compared to hydro-distillation (HD). Influence of microwave power 200-500 watt (W) and irradiation duration (3-5 hours) on essential oil extraction and chemical compounds were analysed via gas chromatography with flame ionization detection (GC-FID) and gas chromatography with mass spectrometry (GC-MS). MAE extracts yield at $500 \mathrm{~W}$ in 5 hours is $0.22 \%$ and the main components present were 4 phenyl-2-butanone (11.5\%), hinesol (10.3\%), and guaiol (5.9\%), while yield of HD extracts in 12 hours is $0.20 \%$ and the major components present were 4-phenyl-2-butanone $(7.0 \%)$, eudesmol $(11.5 \%)$ and hinesol $(9.0 \%)$. The percent composition of chemical compounds from MAE extracts $(87.0 \%)$ and HD extracts $(88.4 \%)$ were quite similar. MAE is more advantageous than HD in terms of energy savings, extraction time ( 5 hours for MAE against 12 hours for HD) and number of identified compounds; MAE identified 57 compounds while 49 compounds by HD. MAE offers a modern, fast and green technology. 